\title{
Analysis of Cantilever Beam Deflection under Uniformly Distributed Load using Artificial Neural Networks
}

\author{
T.M.Y.S Tuan Ya $^{1,{ }^{*}}$, Reza Alebrahim ${ }^{2}$, Nadziim Fitri $^{1}$, and Mahdi Alebrahim ${ }^{3}$ \\ ${ }^{1}$ Department of Mechanical Engineering, Universiti Teknologi PETRONAS, 32610 Seri Iskandar, Perak, Malaysia \\ ${ }^{2}$ High Performance Cloud Computing Centre, Universiti Teknologi PETRONAS, 32610 Seri Iskandar, Perak, Malaysia \\ ${ }^{3}$ Faculty of Mechanical Engineering, Universiti Teknologi Malaysia, Malaysia
}

\begin{abstract}
In this study the deflection of a cantilever beam was simulated under the action of uniformly distributed load. The large deflection of the cantilever beam causes the non-linear behavior of beam. The purpose of this study is to predict the deflection of a cantilever beam using Artificial Neural Networks (ANN). The simulation of the deflection was carried out in MATLAB by using 2-D Finite Element Method (FEM) to collect the training data for the ANN. The predicted data was then verified again through a non linear 2-D geometry problem solver, FEM. Loads in different magnitudes were applied and the non-linear behaviour of the beam was then recorded. It was observed that, there is a close agreement between the predicted data from ANN and the results simulated in the FEM.
\end{abstract}

\section{Introduction}

Deflection in the beam and beam-like structures under external loads has always been important for engineers. To some extent, this does not affect the overall construction, but if the deflection is large enough, it causes instability and the structure becomes insecure. Multiple analytical solutions have been presented by researchers in order to solve large beam deflection. In spite of that, the technique or procedure of developing the analytical models is quite challenging as there is no straightforward analytical solution to simplify the nonlinearity terms presented in the traditional governing equations. Therefore, they have taken different approaches to solve the complex equations related to large deflection of beams by using finite element method (FEM). Consequently, a better and simpler method is needed to reinforce the FEM so there will be less time and effort to be taken in order to develop the parametric studies.

Artificial Neural Networks (ANN) method can be used in building structural planning, in which it is one of the computational modelling that has been developed rapidly along with the development of science (Fig. 1). ANN is an adaptive and self-organized method. Thus, it can change its parameters and network structure based on input data. Data input will be processed to be able to recognize other input data that has not been previously recognized. Processing on ANN is performed using nonlinear activation function. Unlike conventional computational methods that are generally non-adaptive, linear and sequential, these properties of ANN are the basis of a system capable of solving various problems flexibly. Artificial Neural Network is a part of Artificial Intelligence (AI). AI is a huge field of computer engineering operating the system of a machine like how human brain works, which is also called intelligent machine. AI has actually been developed almost over a decade and now it shows great development, especially in engineering field. A few years ago, it was a great problem for engineers in engineering design and material management to solve complicated problems which dealt with many uncertainties.

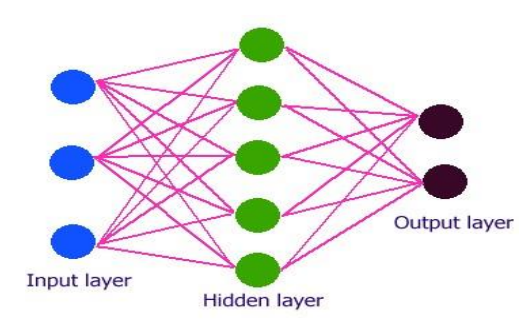

Figure 1. Processing unit ANN feed-forward

The major problem in solid mechanics research on large deflection of cantilever beam is to solve the nonlinear term presented in the governing equation of large deflection of cantilever beam. Thus, many researchers have taken various analytical tools to solve the non-linear problem. Early work by Chen [1], suggested an integral way to solve the problems of large deflection of cantilever beams. Additional work

\footnotetext{
* Corresponding author: tyusoff.ty@utp.edu.my
} 
was done by Pawar and Sawant [2] in static analysis of cantilever beam. He used Bernoulli-Euler relationship of uniform rectangular beam. Later, the numerical results were compared with the ANSYS results. In another research, in order to examine the pattern of large and small deflection of cantilever beams, a study was carried out by Neipp et al. [3] by using the Bernoulli-Euler method. He successfully derived an equation of beam in non-linear terms for both large and small conditions. Interestingly, he obtained very close results in both conditions. Later in 2014, Kimiaeifar et al. [4] took different initiative by using Homotopy Analysis Method (HAM) in solving problems of large deflection analysis of cantilever beam under point load and uniform distributed load. The results from the study were compared with Finite Element Method (FEM). The obtained results were also in good agreement. In addition, back in 2002, a study of analysis of large deflection of cantilever beam under combined loading was done by Lee [5] with the methods of Ludwick relation and Bernoulli-Euler. Later, after getting the equation, he solved it by using one parameter shooting method. It was then compared with G. Lewis's result, which shows close similarity. It can be concluded that solving the problem of deflection of cantilever beam is best using ANN method as all previous researches clearly have shown that the final findings generated from computational method were very accurate and precise.

To predict the deflection of cantilever beams under uniformly distributed loads applied on the beam in the y-direction, an Artificial Neural Networks (ANNs) that may help with the development of strength prediction models was used. Secondly, it was meant to solve the beam selection problem, underlining any difficulty or issue with the algorithm, as it is normally being done with a more traditional statistical technique. Thirdly, the research intends to analyse the results from the FEM simulation and prediction data of ANN in order to prove the accuracy of ANN data prediction.

\section{Research Methodology}

\subsection{Cantilever Beam - Uniformly Distributed Load Structure}

A cantilever beam with distributed load was considered (Fig. 2). Uniform load (w), which acts downwards to the beam, is varied in 50 different loads starting from
$20 \mathrm{~N} / \mathrm{mm}$ to $58.36 \mathrm{~N} / \mathrm{mm}$. Material A-36 steel is used. In this study, 360 data are used for the different loads, ranging from $20 \mathrm{~N} / \mathrm{mm}$ to $58.36 \mathrm{~N} / \mathrm{mm}$. There are 1140 number of elements for the model of cantilever beam simulation.

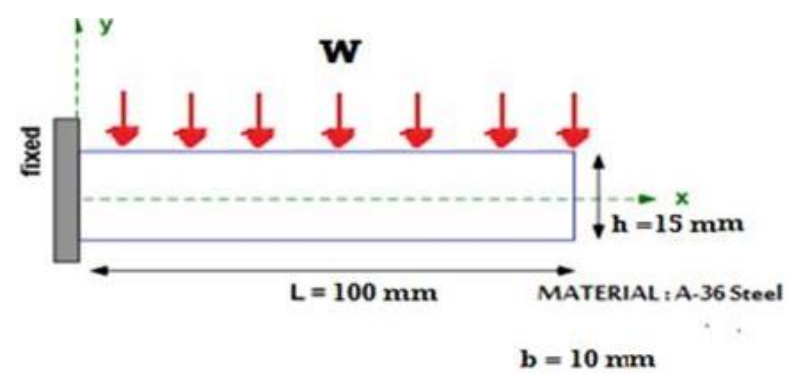

Figure 2. Cantilever beam under distributed load

\subsection{FEM Implementation in MATLAB}

Multiple FEM codes were written on MATLAB in order to simulate the deflection of A-36 Steel cantilever beam with a uniform load acting on top of the beam. The beam is $100 \mathrm{~mm}$ in length, $10 \mathrm{~mm}$-width and $15 \mathrm{~mm}$-height. The modulus of elasticity of the beam is $200 \mathrm{GPa}$ with Poisson's ratio of 0.3. The uniformly distributed loads acting on the beams in the $y$-direction are varied for 50 different loads in the range of $20 \mathrm{~N} / \mathrm{mm}$ to $58.36 \mathrm{~N} / \mathrm{mm}$.

\subsection{Artificial Neural Networking Initialisation}

In the first step, two tables are created for inputs and targets in the workspace window of MATLAB. For inputs table, variable loads or weights were considered while, for the targets table, deflection of beam in both horizontal and vertical directions were tabulated. From the FEM results of 50 different loads, only 40 out of 50 numbers of data (the first 40 loads), which contain 1x360 matrices nodes, were used in the training of ANN. Next, the function of nntool was implimented in the workspace to begin the Neural Network Toolbox. In the training process, a few constraints were set. For example, the number of samples that controls the percentage number of target values. All parameters and values mentioned are listed in Table 1.

Table 1. ANN Neural Fitting

\begin{tabular}{|c|c|c|}
\hline & Target Values & No. of Samples \\
\hline Training & $70 \%$ & 28 \\
\hline Validation & $20 \%$ & 8 \\
\hline Testing & $10 \%$ & 4 \\
\hline
\end{tabular}


For training, Lavenberg-Marquardt Algorithm Method (LMA) was chosen as a training algorithm. LMA is an iterative method that detects the minimum of a multivariate function and is depicted as the sum of squares of non-linear real-valued functions. In fact, it is one of the typical methods used for non-linear least squares problems.

The networks were trained once and the results for every single load were obtained by simply writing the command of function net in the workspace in the MATLAB to begin with the ANN data testing. This step was repeated with 10 sample of loads outside the range of training data sets. The values of loads that were tested in the Neural Network Toolbox are varied from $47.88 \mathrm{~N} / \mathrm{mm}$ to $58.36 \mathrm{~N} / \mathrm{mm}$, which later were compared with the findings from FEM simulation. The comparison data of ANN and FEM are available in the result and discussion section.

In order to get the clear results and detail comparison between both the methods, the collected data of ANN was recorded and translated into simulation by using FEM method. This validation process is similar to loopback test in which inputs are produced by FEM to be utilized in the training process of ANN, and the outputs of ANN are then applied in FEM coding to validate the outputs that are generated by ANN in the form of simulation.

\section{Results and Discussion}

\subsection{Analytical Solution}

By solving the problem analytically, the maximum deflection of the beam was calculated. The maximum deflection was obtained on the free end of the beam, or $\mathrm{x}=100 \mathrm{~mm}$. The deflection for $\mathrm{W}=58.36 \mathrm{~N} / \mathrm{mm}$ is shown in Equation 1.

$$
\delta=\frac{W L^{4}}{8 E I}=-1.297 \mathrm{~mm}
$$

Deflections for different values of loading are tabulated in Table 2.
Table 2. FE deflections for various loadings

\begin{tabular}{|c|c|}
\hline Load (N/mm) & Deflection, $\mathrm{mm}$ \\
\hline 47.88 & -1.064 \\
\hline 49.05 & -1.090 \\
\hline 50.22 & -1.116 \\
\hline 51.39 & -1.142 \\
\hline 52.56 & -1.168 \\
\hline 53.73 & -1.194 \\
\hline 54.90 & -1.220 \\
\hline 56.07 & -1.246 \\
\hline 57.24 & -1.272 \\
\hline 58.36 & -1.297 \\
\hline
\end{tabular}

\subsection{Finite Element Method}

The result of the deflection simulation of cantilever beam analysis using the FEM with the help of the software MATLAB R2014a can be seen in Fig. 3.

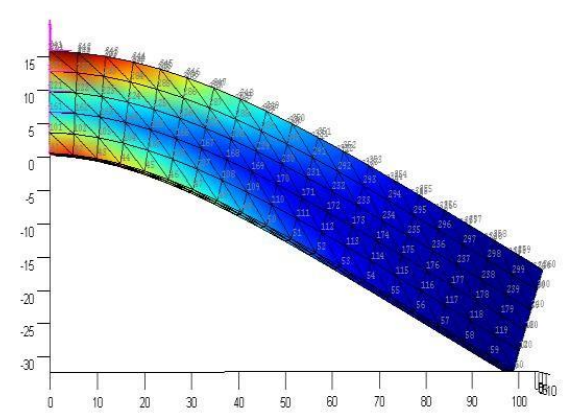

Figure 3. FEM beam simulation under $58.36 \mathrm{~N} / \mathrm{mm}$ uniform load (deflection is 25 times magnified)

To get results with very good level of accuracy (convergent), FEM simulation was done on some modeling with high elements number of 1140 . The higher the number of elements used in FEM simulation, the more precise the results of cantilever beams are.

\subsection{Solving with ANN}

Each network created in the ANN is trained, tested and validated for all samples of data in order to identify the best technique for this study. The data input for the network is used from the simulation beam deflection in FEM and is stored in MS Excel form. The data is distributed into training (70\%), testing $(20 \%)$ and validation (10\%). The percentage of the values stored for each process is determined based on the importance and relevance required. Only 10\% or 4 samples were allocated for testing since it has no 
effect on training while more focus or emphasis is given to training part, which 28 samples were allocated to. The process is continued by using Neural Network Toolbox (MATLAB) in a way to obtain the value of Mean Squared Error (MSE) and regression R, near to 0 and 1 respectively. During the network training phase, rate of learning and the best neuron numbers in the hidden layer were measured. ANN studies the network to diagnose the shape and data distribution of the beam deflection under different loads. After reaching acceptable small variation of error, the neural network training was stopped. Later, Neural Network model was tested, and the results were validated by comparing against $\mathrm{FE}$ simulation. In this study, three different number of hidden layers are kept as variables and tested on two different loads only, $50.22 \mathrm{~N} / \mathrm{mm}$ and $58.36 \mathrm{~N} / \mathrm{mm}$. This method is tested only for $50.22 \mathrm{~N} / \mathrm{mm}$ and $58.36 \mathrm{~N} / \mathrm{mm}$ loads because the main purpose is to clarify the best number of hidden layers that gives very accurate results for the data used. Later, the steps are repeated with the other 8 different loads. Table 4 shows the comparison in term of error percentage between FEM and ANN results for different number of hidden layers ( 3,5 and 10 hidden layers).

Table 4. ANN data for 3,5 and 10 number of hidden layers \& relative error between ANN and FEM results.

\begin{tabular}{|c|c|c|c|c|c|c|c|c|c|c|c|c|}
\hline \multirow{4}{*}{$\begin{array}{l}\text { No. of } \\
\text { Hidden } \\
\text { Layer }\end{array}$} & \multirow{2}{*}{\multicolumn{4}{|c|}{$\begin{array}{c}\text { ANN } \\
\text { Load (N/mm) }\end{array}$}} & \multirow{2}{*}{\multicolumn{4}{|c|}{$\begin{array}{l}\text { FEM } \\
\text { Load (N/mm) }\end{array}$}} & \multicolumn{4}{|c|}{ \% ERROR } \\
\hline & & & & & & & & & & & $(\mathrm{N} / \mathrm{mm})$ & \\
\hline & 50.22 & 58.36 & 50.22 & 58.36 & 50.22 & 58.36 & 50.22 & 58.36 & 50.22 & 58.36 & 50.22 & 58.36 \\
\hline & \multicolumn{2}{|c|}{$\delta x(\mathrm{~mm})$} & \multicolumn{2}{|c|}{$\delta \mathrm{y}(\mathrm{mm})$} & \multicolumn{2}{|c|}{$\delta \mathbf{x}(\mathrm{mm})$} & \multicolumn{2}{|c|}{$\delta y(\mathrm{~mm})$} & \multicolumn{2}{|c|}{$\delta x(m m)$} & \multicolumn{2}{|c|}{$\delta y(\mathrm{~mm})$} \\
\hline 3 & -0.1201 & -0.1396 & -1.1157 & \begin{tabular}{|l|}
1.296 \\
\end{tabular} & \multirow{3}{*}{-0.1200} & \multirow{3}{*}{-0.139} & \multirow{3}{*}{-1.116} & \multirow{3}{*}{-1.297} & 0.0833 & 0.4317 & 0.0269 & 0.0771 \\
\hline 5 & -0.1199 & -0.1399 & \begin{tabular}{|l|l|}
-1.1158 \\
\end{tabular} & -1.2988 & & & & & 0.0833 & 0.6475 & 0.0179 & 0.1388 \\
\hline 10 & -0.1543 & -0.1832 & -1.1743 & -1.6724 & & & & & 28.58 & 31.80 & 5.224 & 28.94 \\
\hline
\end{tabular}

From the Table 4, we can also see that the best number of hidden layers, 3-hidden layers gives the lowest \% error. However, for 5-hidden layers, it depicts low percentage error but slightly higher than 3-hiddenlayer. Results for 10-hidden-layer display a huge difference between the other two numbers of hidden layer. Therefore, it is necessary to use 3-hidden-layer since it has the lowest percentage of error, which is reliable to give the best results at the end of this study.

Next, for the ANN process, the free end displacement was predicted for the different loading where the loads are outside the training range. Finally, the computed results obtained from the ANN were compared and analyzed with the FEM simulation results. The comparison depends on the accuracy and consistency of the solutions or results, and also their effectiveness in interpreting the solid mechanics fundamentals. It is done to check efficiency of prediction of the ANN.
Table 5. Relative error between FEM \& ANN results

\begin{tabular}{|c|c|c|c|c|c|c|c|}
\hline \multirow{2}{*}{$\begin{array}{c}\text { Sample } \\
\text { No. }\end{array}$} & \multirow{2}{*}{$\begin{array}{c}\text { Load } \\
(\mathrm{N} / \mathrm{mm})\end{array}$} & \multicolumn{2}{|c|}{ FEM } & \multicolumn{2}{|c|}{ ANN } & \multicolumn{2}{|c|}{ \% Error } \\
\hline & & $\delta \mathrm{x} / \mathrm{mm}$ & $\delta \mathrm{y} / \mathrm{mm}$ & $\delta \mathrm{x} / \mathrm{mm}$ & $\delta \mathrm{y} / \mathrm{mm}$ & $\delta \mathrm{x}$ & $\delta y$ \\
\hline 1 & 47.88 & -0.1140 & -1.0640 & -0.1143 & -1.0639 & 0.26 & 0.01 \\
\hline 2 & 49.05 & -0.117 & -1.09 & -0.1173 & -1.0898 & 0.26 & 0.02 \\
\hline 3 & 50.22 & -0.120 & -1.116 & -0.1201 & -1.1157 & 0.08 & 0.03 \\
\hline 4 & 51.39 & -0.123 & -1.142 & -0.1239 & -1.1416 & 0.73 & 0.04 \\
\hline 5 & 52.56 & -0.125 & -1.168 & -0.1259 & -1.1675 & 0.71 & 0.04 \\
\hline 6 & 53.73 & -0.128 & -1.194 & -0.1288 & -1.1933 & 0.62 & 0.06 \\
\hline 7 & 54.90 & -0.131 & -1.220 & -0.1317 & -1.2191 & 0.53 & 0.07 \\
\hline 8 & 56.07 & -0.134 & -1.246 & -0.1347 & -1.2448 & 0.52 & 0.10 \\
\hline 9 & 57.24 & -0.137 & -1.272 & -0.1376 & -1.2704 & 0.44 & 0.13 \\
\hline 10 & 58.36 & -0.139 & -1.297 & -0.1396 & -1.296 & 0.43 & 0.08 \\
\hline
\end{tabular}

In general, the deflection results were obtained from the changes in $\mathrm{y}$-direction and $\mathrm{x}$-direction. From Table 5, when the beam is loaded with uniform load of $58.36 \mathrm{~N} / \mathrm{mm}$ acting in the $\mathrm{y}$-direction, the deflection on y-direction obtained to be $1.297 \mathrm{~mm}$ and $0.139 \mathrm{~mm}$ on the $\mathrm{x}$-direction. Meanwhile, the predicted ANN data for $58.36 \mathrm{~N} / \mathrm{mm}$ loading was recorded to be 1.296 $\mathrm{mm}$ and $0.1396 \mathrm{~mm}$ which are very close to the FEM results. Plus, the percentage error between FEM and ANN results of $58.36 \mathrm{~N} / \mathrm{mm}$ are very small with $0.43 \%$ and $0.08 \%$. Overall, from the comparison of the FEM model results with the prediction results of ANN, which is shown in Fig. 4, it can be seen that the relative errors between both techniques for $\mathrm{x}^{-}$ direction deflection are not more than $1 \%$ on average, which are very low and in acceptable range. In addition, in order to validate the results of ANN, a zoomed-in view is shown in the Fig. 5. The result shows that there is a very close agreement between ANN and FEM model and ANN result nearly matches the FEM result, which makes it hard to notice the differences between them in Fig. 5 .

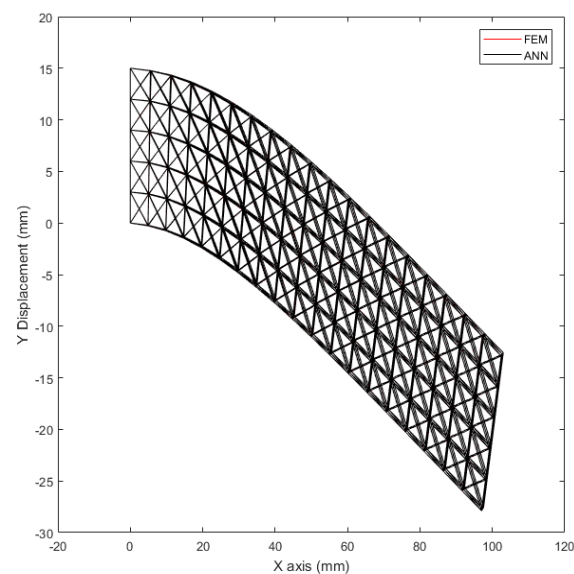

Figure 4. Correlation between ANN \& FEM models for 58.36 $\mathrm{N} / \mathrm{mm}$ load (deflection is 25 times magnified) 


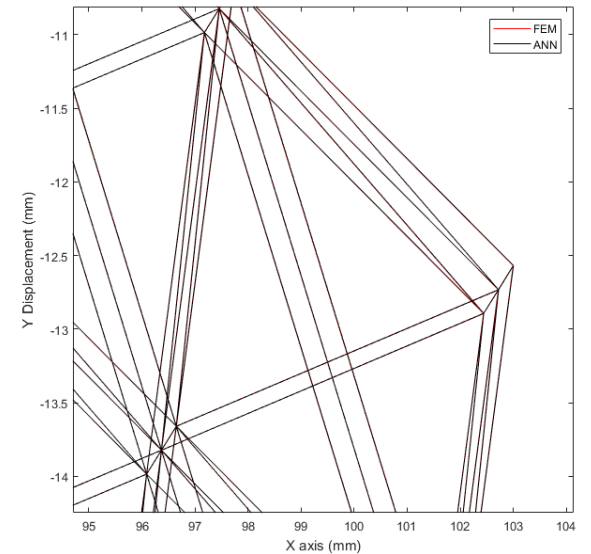

Figure 5. Correlation between ANN \& FEM models for 58.36 $\mathrm{N} / \mathrm{mm}$ load (zoomed in)

\section{Conclusion}

The maximum deflection of a cantilever beam under distributed load was predicted using Artificial Neural Network. Maximum deflection of a cantilever beam was initially calculated through FE simulation. Results were then categorized to train artificial neural networks. It was seen that accurate results can be derived using three hidden layers. It was also observed that ANN can accurately detect the behaviour of 2D cantilever beam. It was concluded Artificial Neural Network is a very effective tool to solve deflection of cantilever beam under uniform loads.

\section{Acknowledgement}

This research is funded by Universiti Teknologi PETRONAS URIF grant 0153AA-G26. The authors are also thankful to HPC3 Universiti Teknologi PETRONAS for their facilities, support and help.

\section{References}

[1] Chen, L. (2010). An integral approach for large deflection cantilever beams. International Journal of NonLinear Mechanics,45(3).

[2] Neipp, C., Beléndez, T., \& Beléndez, A. (2002). Large and small deflections of a cantilever beam. European Journal of Physics,23(3), 371-379.

[3] R.S. Pawar \& S.H. Sawant. (2014). Experimental Static Analysis of a Cantilever Beam with Nonlinear Parameters. InternationalJournal of Engineering Sciences \& Research Technology, ISSN:2277-9655. Retrieved June 13, 2017.
[4] Kimiaeifar, A., Tolou, N., Barari, A., \& Herder, J. (2013). Large deflection analysis of cantilever beam under end point and distributed loads. Journal of the Chinese Institute of Engineers,37(4), 438-445. doi:10.1080/02533839.2013.814991

[5] Lee, K. (2002). Large deflections of cantilever beams of non-linear elastic material under a combined loading. International Journal of Non-Linear Mechanics,37(3), 439-443. doi:10.1016/s0020 - 7462(01)00019-1 